\title{
Editorial
}

\section{From Association to Function in the Post-GWAS Era}

\author{
Takanari Gotoda
}

Department of Biochemistry, Faculty of Medicine, Kyorin University, Tokyo, Japan

Key words: Genome-wide association, Lipid, SNP, QTL

\section{See article vol. 22: $455-480$}

During the past decade, the genome-wide association studies (GWAS) have revolutionized the world of genetics by their enormous power to comprehensively detect genetic association between gene variants (e.g., SNPs) and complex phenotypes (e.g., quantitative traits or diseases). One of the most successful examples was the identification of gene loci associated with blood lipid levels in humans ${ }^{1,2}$. The original findings obtained by a large global consortium were quite reproducible and consistent with results of other studies $^{3)}$, implying blood lipids as highly reliable quantitative traits.

GWAS could provide a comprehensive collection of genomic variants of significant association, together with their individual chromosomal locations at the genome-wide significance level. However, GWAS themselves have only limited power to pinpoint the causative gene(s) or gene mutation(s) that underlie the complex trait (or disease) of interest. In most cases, such genomic variants with the strongest association signal (i.e., lead SNPs) are located outside the coding region of the gene; thus, providing little evidence in terms of their functional significance, whereas some of these variants are believed to affect the expression of the nearby (cis) or distant (trans) gene(s) either via direct or indirect mechanism. In general, subsequently to GWAS, several complimentary approaches have been attempted to explore and establish the causative genes, such as physical fine-mapping approach to narrow down the candidate genomic region, expression quan-

Address for correspondence: Takanari Gotoda, Department of Biochemistry, Faculty of Medicine, Kyorin University, 6-20-2

Shinkawa, Mitaka-city, Tokyo, 181-8611, Japan

E-mail: gotoda@ks.kyorin-u.ac.jp

Received: April 14, 2015

Accepted for publication: April 15, 2015 titative trait loci (eQTL) analysis to explore association between gene variants and mRNA expression levels, bioinformatics analysis in consideration with metabolic pathways and their interaction according to available databases, and functional validation approach to establish the functional significance with in vitro and/or in vivo biological experiments (Fig. 1). In spite of such attempts, demonstration of the causative gene/ mutation has been quite difficult and remains very challenging. Although GWAS had revealed that sortilin (SORT1) was the novel target gene with the third strongest signal for association with blood low-density lipoprotein (LDL) cholesterol levels ${ }^{1)}$, functional validation approaches with overexpression or knock-down experiments have produced conflicting and inconsistent results ${ }^{4)}$. Thus, the experimental strategy after GWAS has not been so straightforward, and application of an additional approach will be eagerly encouraged.

On page 455 of this issue of Journal of Atherosclerosis and Thrombosis, Akiyama et al. reports a unique complimentary approach to search for functional genes responsible for positive association signals revealed by GWAS ${ }^{5)}$. First, the authors have selected rat genes orthologous to human candidate genes revealed by the lipid GWAS mentioned above ${ }^{1,2)}$ and subsequently have examined in vivo alterations in the mRNA expression levels of such candidate genes secondary to the intervention by feeding rats with highfat and high-cholesterol diets (HFD) or with administration of a cholesterol-lowering agent, statin. With intervention by HFD, seven known, established candidate genes have been selected, giving authenticity to the rationality of the study design, and three novel significant and 23 additional suggestive genes have also been identified. Among them, Stac3 appears to be an interesting candidate target of lipid metabolism because it was the only novel gene that was regulated in response to both HFD and statin treatment, with the stringent and nominal levels of statistical signifi- 
Genome-wide association scans using polymorphic markers (e.g., SNPs)
Physical fine mapping to narrow down the candidate region Investigation based on data derived from eQTL analyses

Reference to the available databases to annotate genetic variants
Integration of in vitro and in vivo experimental approaches to validate the functional significance
Identification of association of gene loci by GWAS
Fine mapping for causative genes or variants
Functional validation

Position

Target genes

Function

Fig. 1. Schematic overview of the experimental strategy from association to functional validation.

Gene loci identified by genome-wide association scans undergo further scrutiny by physical fine-mapping, expression quantitative trait loci (eQTL) analyses, and references to the available databases. Demonstration of the functional significance of the causative variants requires in vitro and/or in vivo functional validation approaches.

cance, respectively. Although the expression of Stac3 is known to be most abundant in skeletal muscles and nutritionally regulated ${ }^{6}$, there has been no direct evidence published to suggest possible involvement of Stac3 in lipid metabolism. On the basis of the data presented by Akiyama et al. in the current issue, further investigation is therefore warranted to examine such involvement.

Another impressive finding came from a comparison between the current results reported by Akiyama et al. and the previously published results derived from cis-eQTL analyses and/or from RNAibased functional profiling ${ }^{1,7)}$. Despite partial overlaps observed between candidate genes that were detected by each approach, Lipg was the only gene commonly detectable by all three independent approaches. This finding highlights the importance of application of different experimental approaches to avoid missing crucial genes owing to reliance on a single approach alone as well as to enrich the collection of target genes of GWAS association more systematically.

Inconsistent with the brilliant results provided by GWAS, their application to clinical diagnosis or therapeutic research has been very limited. In addition, they have rarely provided mechanistic insights. In this regard, the unique experimental approach described by Akiyama et al. would be of potential use and might be regarded as "functional fine-mapping" because it combines the gene mapping approach with the in vivo functional evaluation approach performed in an animal model. Development of such diverse effective approaches will be highly encouraged to fill the great gap between association and function still left in the post-GWAS era.

\section{Conflicts of Interest}

None.

\section{References}

1) Teslovich TM, Musunuru K, Smith AV, Edmondson AC, Stylianou IM, Koseki M, Pirruccello JP, Ripatti S, Chasman DI, Willer CJ, Johansen CT, Fouchier SW, Isaacs A, Peloso GM, Barbalic M, Ricketts SL, Bis JC, Aulchenko YS, Thorleifsson G, Feitosa MF, Chambers J, OrhoMelander M, Melander O, Johnson T, Li X, Guo X, Li M, Shin Cho Y, Jin Go M, Jin Kim Y, Lee JY, Park T, Kim K, Sim X, Twee-Hee Ong R, Croteau-Chonka DC, Lange LA, Smith JD, Song K, Hua Zhao J, Yuan X, Luan J,Lamina C, Ziegler A, Zhang W, Zee RY, Wright AF, Witteman JC, Wilson JF, Willemsen G, Wichmann HE, Whitfield JB, Waterworth DM, Wareham NJ, Waeber G, Vollenweider P, Voight BF, Vitart V, Uitterlinden AG, Uda M, Tuomilehto J, Thompson JR, Tanaka T, Surakka I, Stringham HM, Spector TD, Soranzo N, Smit JH, Sinisalo J, Silander K, Sijbrands EJ, Scuteri A, Scott J, Schlessinger D, Sanna S, Salomaa V, Saharinen J, Sabatti C, Ruokonen A, Rudan I, Rose LM, Roberts R, Rieder M, Psaty BM, Pramstaller PP, Pichler I, Perola M, Penninx BW, Pedersen NL, Pattaro C, Parker AN, Pare G, Oostra BA, O’Donnell CJ, Nieminen MS, Nickerson DA, Montgomery GW, Meitinger T, McPherson R, McCarthy MI, McArdle W, Masson D, Martin NG, Marroni F, Mangino M, Magnusson PK, Lucas G, Luben R, Loos RJ, Lokki ML, Lettre G, Langenberg C, Launer LJ, Lakatta EG, Laaksonen R, Kyvik KO, Kronenberg F, König IR, Khaw KT, Kaprio J, Kaplan LM, Johansson A, Jarvelin MR, Janssens AC, Ingelsson E, Igl W, Kees Hovingh G, Hottenga JJ, Hofman A, Hicks AA, Hengstenberg C, Heid IM, Hayward C, Havulinna AS, Hastie ND, Harris TB, Haritunians T, Hall AS, Gyllensten U, Guiducci C, Groop LC, Gonzalez E, Gieger C, Freimer NB, Ferrucci L, Erdmann J, Elliott P, Ejebe KG, Döring A, Dominiczak AF, Demissie S, Deloukas P, de Geus EJ, de Faire U, Crawford G, Collins FS, Chen YD, Caulfield MJ, Campbell H, Burtt NP, Bonnycastle LL, Boomsma DI, Boekholdt SM, Bergman RN, Barroso I, Bandinelli S, Ballantyne CM, Assimes TL, Quertermous T, Altshuler D, Seielstad M, Wong TY, Tai ES, Feranil AB, Kuzawa CW, Adair LS, Taylor HA Jr, Borecki I 1 B, Gabriel SB, Wilson JG, Holm H, Thorsteinsdottir U, Gudnason V, Krauss RM, Mohlke KL, Ordovas JM, Munroe PB, Kooner JS, Tall AR, Hegele RA, Kastelein JJ, Schadt EE, 
Rotter JI, Boerwinkle E, Strachan DP, Mooser V, Stefansson K, Reilly MP, Samani NJ, Schunkert H, Cupples LA, Sandhu MS, Ridker PM, Rader DJ, van Duijn CM, Peltonen L, Abecasis GR, Boehnke M, Kathiresan S: Biological, clinical and population relevance of 95 loci for blood lipids. Nature, 2010; 466: 707-713

2) Global Lipids Genetics Consortium, Willer CJ, Schmidt EM, Sengupta S, Peloso GM, Gustafsson S, Kanoni S, Ganna A, Chen J, Buchkovich ML, Mora S, Beckmann JS, Bragg-Gresham JL, Chang HY, Demirkan A, Den Hertog HM, Do R, Donnelly LA, Ehret GB, Esko T, Feitosa MF, Ferreira T, Fischer K, Fontanillas P, Fraser RM, Freitag DF, Gurdasani D, Heikkilä K, Hyppönen E, Isaacs A, Jackson AU, Johansson A, Johnson T, Kaakinen M, Kettunen J, Kleber ME, Li X, Luan J, Lyytikäinen LP, Magnusson PK, Mangino M, Mihailov E, Montasser ME, Müller-Nurasyid M, Nolte IM, O'Connell JR, Palmer CD, Perola M, Petersen AK, Sanna S, Saxena R, Service SK, Shah S, Shungin D, Sidore C, Song C, Strawbridge RJ, Surakka I, Tanaka T, Teslovich TM, Thorleifsson G, Van den Herik EG, Voight BF, Volcik KA, Waite LL, Wong A, Wu Y, Zhang W, Absher D, Asiki G, Barroso I, Been LF, Bolton JL, Bonnycastle LL, Brambilla P, Burnett MS, Cesana G, Dimitriou M, Doney AS, Döring A, Elliott P, Epstein SE, Eyjolfsson GI, Gigante B, Goodarzi MO, Grallert H, Gravito ML, Groves CJ, Hallmans G, Hartikainen AL, Hayward C, Hernandez D, Hicks AA, Holm H, Hung YJ, Illig T, Jones MR, Kaleebu P, Kastelein JJ, Khaw KT, Kim E, Klopp N, Komulainen P, Kumari M, Langenberg C, Lehtimäki T, Lin SY, Lindström J, Loos RJ, Mach F, McArdle WL, Meisinger C, Mitchell BD, Müller G, Nagaraja R, Narisu N, Nieminen TV, Nsubuga RN, Olafsson I, Ong KK, Palotie A, Papamarkou T, Pomilla C, Pouta A, Rader DJ, Reilly MP, Ridker PM, Rivadeneira F, Rudan I, Ruokonen A, Samani N, Scharnagl H, Seeley J, Silander K, Stancáková A, Stirrups K, Swift AJ, Tiret L, Uitterlinden AG, van Pelt LJ, Vedantam S, Wainwright N, Wijmenga C, Wild SH, Willemsen G, Wilsgaard T, Wilson JF, Young EH, Zhao JH, Adair LS, Arveiler D, Assimes TL, Bandinelli S, Bennett F, Bochud M, Boehm BO, Boomsma DI, Borecki IB, Bornstein SR, Bovet P, Burnier M, Campbell H, Chakravarti A, Chambers JC, Chen YD, Collins FS, Cooper RS, Danesh J, Dedoussis G, de Faire U, Feranil AB, Ferrières J, Ferrucci L, Freimer NB, Gieger C, Groop LC, Gudnason V, Gyllensten U, Hamsten A, Harris TB, Hingorani A, Hirschhorn JN, Hofman A, Hovingh GK, Hsiung CA, Humphries SE, Hunt SC, Hveem K, Iribarren C, Järvelin MR, Jula A, Kähönen M, Kaprio J, Kesäniemi A, Kivimaki M, Kooner JS, Koudstaal PJ, Krauss RM, Kuh D,
Kuusisto J, Kyvik KO, Laakso M, Lakka TA, Lind L, Lindgren CM, Martin NG, März W, McCarthy MI, McKenzie CA, Meneton P, Metspalu A, Moilanen L, Morris AD, Munroe PB, Njølstad I, Pedersen NL, Power C, Pramstaller PP, Price JF, Psaty BM, Quertermous T, Rauramaa R, Saleheen D, Salomaa V, Sanghera DK, Saramies J, Schwarz PE, Sheu WH, Shuldiner AR, Siegbahn A, Spector TD, Stefansson K, Strachan DP, Tayo BO, Tremoli E, Tuomilehto J, Uusitupa M, van Duijn CM, Vollenweider P, Wallentin L, Wareham NJ, Whitfield JB, Wolffenbuttel BH, Ordovas JM, Boerwinkle E, Palmer CN, Thorsteinsdottir U, Chasman DI, Rotter JI, Franks PW, Ripatti S, Cupples LA, Sandhu MS, Rich SS, Boehnke M, Deloukas P, Kathiresan S, Mohlke KL, Ingelsson E, Abecasis GR: Discovery and refinement of loci associated with lipid levels. Nat Genet, 2013; 45: 1274-1283

3) Waterworth DM, Ricketts SL, Song K, Chen L, Zhao JH, Ripatti S, Aulchenko YS, Zhang W, Yuan X, Lim N, Luan J, Ashford S, Wheeler E, Young EH, Hadley D, Thompson JR, Braund PS, Johnson T, Struchalin M, Surakka I, Luben R, Khaw KT, Rodwell SA, Loos RJ, Boekholdt SM, Inouye M, Deloukas P, Elliott P, Schlessinger D, Sanna S, Scuteri A, Jackson A, Mohlke KL, Tuomilehto J, Roberts R, Stewart A, Kesäniemi YA, Mahley RW, Grundy SM; Wellcome Trust Case Control Consortium, McArdle W, Cardon L, Waeber G, Vollenweider P, Chambers JC, Boehnke M, Abecasis GR, Salomaa V, Järvelin MR, Ruokonen A, Barroso I, Epstein SE, Hakonarson $\mathrm{HH}$, Rader DJ, Reilly MP, Witteman JC, Hall AS, Samani NJ, Strachan DP, Barter P, van Duijn CM, Kooner JS, Peltonen L, Wareham NJ, McPherson R, Mooser V, Sandhu MS: Genetic variants influencing circulating lipid levels and risk of coronary disease. Arterioscler Thromb Vasc Biol, 2010; 30: 2264-2276

4) Strong A, Rader DJ: Sortilin as a regulator of lipoprotein metabolism. Curr Atheroscler Rep, 2012; 14: 211-218

5) Akiyama $K$, Liang $Y$, Isono $M$, Kato $N$ : Investigation of functional genes in vivo through high-fat-1 diet intervention in rats at homologous loci from blood lipid genomewide association studies. J Atheroscler Thromb, 2015; 22: 455-480

6) Bower NI, de la Serrana DG, Cole NJ, Hollway GE, Lee HT, Assinder S, Johnston IA: Stac3 is required for myotube formation and myogenic differentiation in vertebrate skeletal muscle. J Biol Chem, 2012; 287: 43936-43949

7) Blattmann P, Schuberth C, Pepperkok R, Runz H: RNAibased functional profiling of loci from blood lipid genomewide association studies identifies genes with cholesterolregulatory function. PLoS Genet, 2013; 9: e1003338 